\title{
Interference Mitigation and Sum Rate Optimization for MIMO Downlink Small Cells
}

\author{
Ha Hoang KHA \\ Faculty of Electrical and Electronics Engineering, \\ Ho Chi Minh City University of Technology, VNU-HCM, Vietnam \\ hhkha@hcmut.edu.vn
}

Manuscript received June 06, 2016

\begin{abstract}
This paper addresses interference issues in multiuser multiple-input multiple-output (MIMO) downlink heterogeneous networks in which multiple small cells are deployed in macrocell coverage. With the higher priority to access the frequency bands, the macro base station (MBS) will exploit eigenmode transmission along with water-filling based power allocation to maximize its data rate. To avoid harmful interference to macro users, we propose structures of the precoders at the small cell BSs (SBSs) as cascades of two precoding matrices. In addition, to mitigate intra-tier inference in small cells, the SBSs exploit the user scheduling schemes for their associated users. We investigate two user scheduling schemes using the minimum interference leakage and maximum signal to noise ratio criteria. The sum rate of the selected users can be further improved by power allocation. We develop an iterative algorithm using the difference of convex functions (d.c.) programming to tackle the mathematical challenges of the nonconvex power allocation problem, Numerical simulation results show that the proposed strategy outperforms the conventional methods in terms of the achievable sum rate.
\end{abstract}

\section{Keywords}

MU-MIMO interference channels, interference alignment, small cells, precoder design

\section{Introduction}

The small cell deployment in macrocell coverage has attracted considerable attentions of both academia and industry due to its potential to cope with the increasing user numbers and demand of the mobile traffic in wireless cellular networks [1-4]. The wireless networks in which small cells such as picocells or femtocells are deployed at traffic demanding hotspots or at coverage holes are known as heterogeneous networks (HetNets). HetNets have been introduced in Generation Partnership Project (3GPP) Long Term Evolution (LTE) since they can offer great potential to offload the traffic, enhance channel capacity and extend coverage in cellular networks [3], [4]. Due to the scarcity of spectrum resources, the small cells are typically designed to reuse the frequency bands of the macrocells. Such an overlaid cell deployment may lead to co-channel interference [4], [5]. Due to simultaneous transmission of multiple cells, HetNets may suffer from interference caused by the cells in the same tier (co-tier interference) and from the cells in different tiers (cross-tier interference) [6]. Then, the system performance can be significantly degraded if interference is not properly managed. Therefore, interference mitigation in HetNets is a crucial issue.

The present paper is concerned with the multiple-input multiple-output (MIMO) downlink HetNets in which one macro base station (MBS) coexists with multiple small cell base stations (SBSs). It is worth noting that such a system model is similar to that in [1], [6], however, the models in [1], [6] are restricted to one user equipment (UE) per cell. Herein, we consider the scenario that there are multiple UEs per cell and the user scheduling is carried out at the BSs. In addition, we introduce the hierarchical transmission strategy for the MBS and SBSs to efficiently mitigate both cros-tier and co-tier interference.

\subsection{Related Works}

There have been intensive research efforts devoted to interference mitigation in HetNets. The conventional methods are interference avoidance and resource partition in time, frequency or space [1], [8]. Such transmission techniques can be time division multiple access (TDMA), frequency division multiple access (FDMA), or space division multiple access (SDMA). These approaches cannot achieve channel capacity. Recently, interference alignment (IA) has been recognized as an efficient scheme to address interference issues and to achieve the optimal degrees-of-freedom (DoFs) in interference networks [7-10]. IA has been widely used to design the transceivers for various wireless communications [11-14]. References [11-13] used the conventional IA schemes to design the precoders and receive filters for $K$-user interference channels. Specifically, references [11], [12] aimed at improving the secrecy rate of $K$-user channels while [13] focused on maximizing the energy efficiency of the secure 
communication systems. Recently, the authors in [14] have considered the MIMO-OFDM HetNets in which single user per cell was considered. They proposed the methods using space-frequency block codes and IA to mitigate interference in the HetNets with various levels of inter-system coordination. On the other hand, the authors in [15] investigated IA for a two-cell network in which each user sends one data stream. Different from the previous studies in [11-15], our paper focuses on the user scheduling schemes and power allocation to mitigate interference in the HetNets.

The general solutions for IA schemes must be found by iterative algorithms. However, the conventional IA schemes require a large amount of computation and extensive CSI of all links and, therefore, make them difficult to apply in cellular networks [10]. Alternatively, opportunistic IA (OIA) techniques have been recently studied in the literature; see, for example [16], [17]. The key idea of OIA is that at each time slot only the user which has the best performance metric can access the spectrum. As compared to conventional IA, OIA is more simple for implementation since it requires only local CSI and can align interference without iterative computation. Authors in [18] studied OIA techniques for uplink MIMO multi-cells in homogeneous networks. A user scheduling strategy for multi-cell uplink networks was investigated in [9]. However, the BS transmits only one data stream to its scheduled user, and homogeneous wireless networks are assumed. For HetNets, the authors in [5], [8] designed the precoders for a hierarchial IA scheme for HetNets. The closed form expressions are found for the systems in which a macrocell with two users coexists with two picocells having one user per cell. The method in therein cannot be extended to the scenarios with the arbitrary number of small cells.

\subsection{Contributions of This Work}

Our goal is to design the transmission strategies of the MBS and SBSs to efficiently mitigate interference and to maximize the sum rate of the network. We propose a hierarchical transmission strategy in which the MBS is given higher priority to access the spectrum than the SBSs. Our strategy is that the MBS employs eigenmode transmission and adopts the water-filling scheme for power allocation to maximize its rate. To prevent interference from the SBSs to the macrocell UE (MUE), the SBSs will align their transmitted signals into unused spatial dimensions at the MUE [1], [19]. Thus, we design the SBS precoders as a cascade of two precoders. One precoder is used to cancel interference from the SBSs to the MUE while the other unitary precoder is generated according to the isotropic distribution [9]. Since SBSs use space-division multiple access, apart from cross-tier interference, co-tier interference will adversely affect the system performance if multiple small cell UEs (SUEs) in the same cell transmit at the same time. Therefore, to cope with interuser interference, the SBSs will invoke the opportunistic user selection. We propose to use two scheduling metrics for the user scheduling. First, the SBS will select the user that can best align interference from all other BSs. Second, the maximum signal to noise ratio (SNR) metric is used to select the user. It is important to remark that although close in notation to the conventional IA schemes [11-13], the current paper distinguishes itself in the following aspects. First, conventional IA would be inefficient to the user scheduling scheme since it requires the global CSI of all users in the networks and performs an exhaustive search of all possible selected users. For each possible set of the selected users, the conventional IA schemes require to conduct iterative algorithms to find the precoding and postcoding matrices, which may result in a prohibitive computational complexity. In contrast, to select the users and mitigate interference, our scheme based on OIA requires solely the local CSI and non-iterative procedures [20]. In addition, if there is a coordination between SBSs, the sum rate of the network can be improved by appropriate power allocation at each SBS. Thus, with the assumption of coordinated SBSs sharing CSI of the selected users, we further optimize the sum rate of the scheduled users. We seek the optimal power allocation (OPA) strategies to maximize the sum rate of the SUEs subject to the power constraint per SBS. The power allocation problem is mathematically challenging due to its non-convexity nature. We develop an iterative algorithm by expressing the objective function as a difference of two convex functions (d.c.) and, then introduce an iterative d.c. algorithm with the convergence guaranteed. The simulation results will be provided to demonstrate the effectiveness of the proposed method.

The rest of the paper is organized as follows. Section 2 describes the small cell wireless networks considered in the paper. In Sec. 3, we introduce the transmission strategies of the MBS and SBSs. Then, optimal power allocation to further improve the sum-rate of small cells using d.c. programming is derived. Simulation results are provided in Sec. 4. Finally, Sec. 5 concludes the paper.

Notations: Matrices and column vectors are represented by boldface capital and lowercase letters, respectively. $\boldsymbol{X}^{T}$ and $\boldsymbol{X}^{H}$ are the transposition and conjugate transposition of matrix $\boldsymbol{X}$, respectively. $\|\boldsymbol{x}\|_{2}$ denotes the Euclidean norm while $\|\boldsymbol{X}\|_{F}$ is the Frobenius norm. $\boldsymbol{I}$ and $\mathbf{0}$ stand for identity and zero matrices with the appropriate dimensions. 〈.>, |. $\mid$ and $\mathbb{E}($.$) are the trace, determinant and expectation op-$ erators, respectively. $\operatorname{col}_{\ell}^{k}(\boldsymbol{X})$ is a matrix whose columns are the $\ell$-th column to $k$-th column of matrix $\boldsymbol{X}$. We define $\langle\boldsymbol{X}, \boldsymbol{Y}\rangle=\operatorname{Real}\left(\left\langle\boldsymbol{X}^{H} \boldsymbol{Y}\right\rangle\right)$. A complex Gaussian random vector with mean $\overline{\boldsymbol{X}}$ and covariance $\boldsymbol{R}_{\boldsymbol{x}}$ is represented by $\boldsymbol{x} \sim \mathcal{C N}\left(\overline{\boldsymbol{x}}, \boldsymbol{R}_{\boldsymbol{x}}\right)$.

\section{System Model}

Consider the downlink of two-tier cellular wireless networks in which $L$ SBSs coexist to share the common frequency resource in the coverage of an MBS, as illustrated in Fig. 1. The MBS, namely BS 0 , is equipped with $N_{0}$ while 


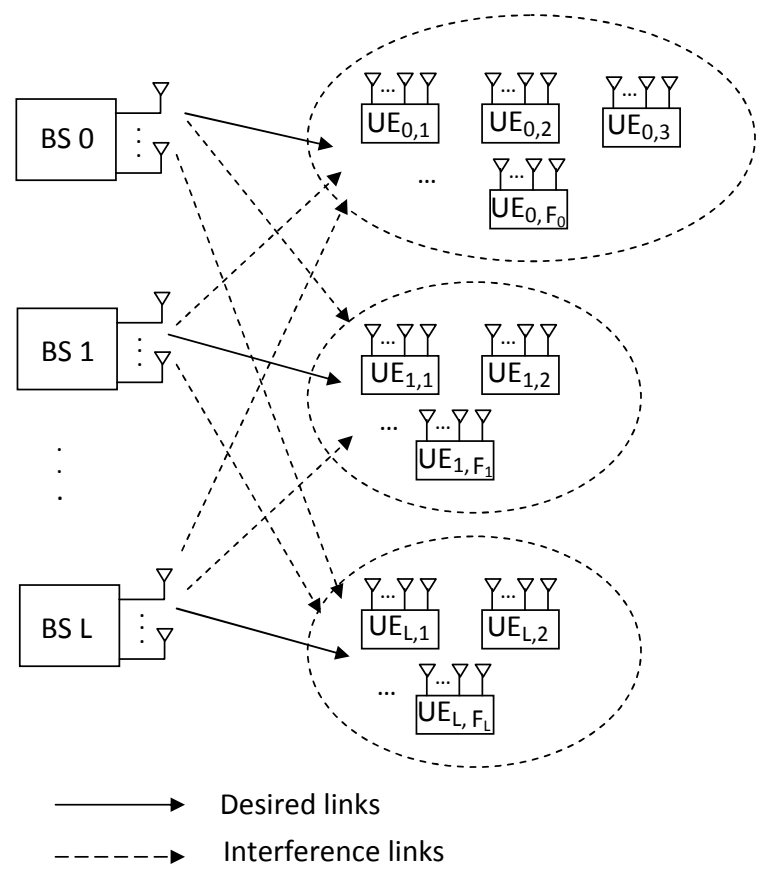

Fig. 1. The system model of the downlink MIMO HetNets.

the SBS denoted by BS $\ell, \ell \in \mathcal{L}=\{1,2, \ldots, L\}$, is equipped with $N_{\ell}$ transmit antennas. Assume that each BS is providing services for multiple UEs. Let $\mathcal{F}_{\ell}$ be a set of users served by BS $\ell$. Consider the scenario of interference coordination in which each user is served by only one BS, i.e., $\mathcal{F}_{i} \cap \mathcal{F}_{j}=\emptyset$ if $i \neq j$. The number of UEs served by $\mathrm{BS} \ell$ is $\left|\mathcal{F}_{\ell}\right|=F_{\ell}$. $\mathrm{UE} k$ in cell $\ell$ is referred to as $\mathrm{UE}_{\ell, k}$. $\mathrm{UE}_{\ell, k}$ is equipped with $M_{\ell, k}$ antennas. At each time slot, each BS selects one UE in its coverage range for transmission [21].

Let $\boldsymbol{x}_{\ell, k} \in C^{d_{\ell, k} \times 1}$ be $d_{\ell, k}$ independent data streams to be transmitted from the $\ell$-th BS to the $k$-th user in cell $\ell$, with $d_{\ell, k} \leq \min \left\{N_{\ell}, M_{\ell, k}\right\} \forall k, \ell$. Define the covariance matrix of the transmitted signals as $\mathbb{E}\left[\boldsymbol{x}_{\ell, k} \boldsymbol{x}_{\ell, k}^{H}\right]=\boldsymbol{Q}_{\ell, k}$. Note that the covariance matrix $\boldsymbol{Q}_{\ell, k}$ is not necessarily diagonal [7]. The $\ell$-th BS, $\ell=0,1, \ldots L$, uses the precoder $V_{\ell, k} \in C^{N_{\ell} \times d_{\ell, k}}$ to map signal $\boldsymbol{x}_{\ell, k}$ into the $N_{\ell}$-dimensional signal to be transmitted over $N_{\ell}$ antennas. Assume that the MIMO channels of the links are block fading, i.e., the channel coefficients remain constant in a time slot and independently vary from one slot to the other. The MIMO channel matrix from the $i$-th BS to the $k$-th user in the $\ell$-th cell is denoted by $\boldsymbol{H}_{\ell, k, i} \in C^{M_{\ell, k} \times N_{i}}$. All local CSI at all BSs can be obtained by using the feedback from the users to its associated BS [19], [22].

At each time slot, one user per cell is selected to communicate with its associated BS ${ }^{1}$. We denote the selected user in cell $\ell$ by $\pi(\ell)$. The received signal $\boldsymbol{y}_{\ell, \pi(\ell)} \in C^{M_{\ell, k} \times 1}$ at selected user $\pi(\ell)$ in cell $\ell$ is given by

$$
\begin{aligned}
\boldsymbol{y}_{\ell, \pi(\ell)}= & \boldsymbol{H}_{\ell, \pi(\ell), \ell} \boldsymbol{V}_{\ell, \pi(\ell)} \boldsymbol{x}_{\ell, \pi(\ell)} \\
& +\sum_{i=0, i \neq \ell}^{L} \boldsymbol{H}_{\ell, \pi(\ell), i} \boldsymbol{V}_{i, \pi(i)} \boldsymbol{x}_{i, \pi(i)}+z_{\ell, \pi(\ell)}
\end{aligned}
$$

where $z_{\ell, k} \in C^{M_{\ell, k} \times 1}$ is additive white Gaussian noise at the $k$-th user in the $\ell$-th cell with $z_{\ell, k} \sim \mathcal{C N}\left(0, \sigma_{\ell, k}^{2} I_{M_{\ell, k}}\right)$. In (1), the first term is the desired signal of the $\pi(\ell)$-th user in the $\ell$-th cell, the second term is inter-cell interference from other cells and the last term is noise. Consider that the $k$-th user in cell $\ell$ uses the postprocessing matrix $\boldsymbol{U}_{\ell, k} \in C^{M_{\ell, k} \times d_{\ell, k}}$ to extract its desired signal transmitted by the corresponding BS $\ell$. After linear processing, the received signal at the admitted user in cell $\ell$ is given by

$$
\begin{aligned}
\boldsymbol{r}_{\ell, \pi(\ell)}= & \boldsymbol{U}_{\ell, \pi(\ell)}^{H} \boldsymbol{y}_{\ell, \pi(\ell)}=\boldsymbol{U}_{\ell, \pi(\ell)}^{H} \boldsymbol{H}_{\ell, \pi(\ell), \ell} \boldsymbol{V}_{\ell, \pi(\ell)} \boldsymbol{x}_{\ell, \pi(\ell)} \\
& +\sum_{i=0, i \neq \ell}^{L} \boldsymbol{U}_{\ell, \pi(\ell)}^{H} \boldsymbol{H}_{\ell, \pi(\ell), i} \boldsymbol{V}_{i, \pi(i)} \boldsymbol{x}_{i, \pi(i)}+\boldsymbol{U}_{\ell, \pi(\ell)}^{H} \boldsymbol{z}_{\ell, \pi(\ell) .}
\end{aligned}
$$

Assuming that Gaussian signalling is employed and interference from other cells is treated as noise, the rate of the $\pi(\ell)$-th user in the $\ell$-th cell is computed by

$$
\begin{aligned}
\mathcal{R}_{\ell, \pi(\ell)}= & \log _{2} \mid \boldsymbol{I}_{d_{\ell, \pi(\ell)}}+\boldsymbol{U}_{\ell, \pi(\ell)}^{H} \boldsymbol{H}_{\ell, \pi(\ell), \ell} \boldsymbol{V}_{\ell, \pi(\ell)} \\
& \times \boldsymbol{Q}_{\ell, \pi(\ell)} \boldsymbol{V}_{\ell, \pi(\ell)}^{H} \boldsymbol{H}_{\ell, \pi(\ell), \ell}^{H} \boldsymbol{U}_{\ell, \pi(\ell)} \boldsymbol{R}_{\ell, \pi(\ell)}^{-1} \mid
\end{aligned}
$$

where

$$
\begin{gathered}
\boldsymbol{R}_{\ell, \pi(\ell)}=\sigma_{\ell, \pi(\ell)}^{2} \boldsymbol{I}_{d_{\ell, \pi(\ell)}} \\
+\sum_{i=0, i \neq \ell}^{L}\left(\boldsymbol{U}_{\ell, \pi(\ell)}^{H} \boldsymbol{H}_{\ell, \pi(\ell), i} \boldsymbol{V}_{i, \pi(i)} \boldsymbol{Q}_{i, \pi(i)} \boldsymbol{V}_{i, \pi(i)}^{H} \boldsymbol{H}_{\ell, \pi(\ell), i}^{H} \boldsymbol{U}_{\ell, \pi(\ell)}\right)
\end{gathered}
$$

is the covariance matrix of the interference-plus-noise at user $\pi(\ell)$ in cell $\ell$.

The major concern in deploying small cell networks is to deal with interference such that the total sum rate of the network is maximized. It is well-known that the design problem of the sum rate maximization is difficult to solve since the achievable rates of the users are interdependent and the associated optimization problem is nonconvex [25]. In next section, we will present transmission strategies to address interference and to maximize the sum rate.

\section{Design Strategies}

Our approach aims at first deriving the MBS transmission strategy and then designing the small cell transmission strategies to efficiently mitigate cross-tier and co-tier interference. Finally, the power allocation algorithm based on d.c. programming is developed to further improve the sum-rate.

\subsection{Macrocell Transmission Strategy}

Provided that the MBS has higher priority to utilize the spectrum [1], [19], the MBS selfishly designs its transmission strategy to maximize its own rate without awareness of the small cell strategies. Since our proposed strategy is to guarantee no cross-tier interference to the MUE and, thus, the MBS can use the channel gain as the scheduling metric [26]. The selected user is computed by

$$
\pi(0)=\arg \max _{k \in \mathcal{F}_{0}}\left\langle\boldsymbol{H}_{0, k, 0} \boldsymbol{H}_{0, k, 0}^{H}\right\rangle .
$$

${ }^{1}$ Note that each BS can serve multiple users at each time slot by allocating orthogonal resources (e.g., OFDMA) to different users [23], [24] 
Concerning cross-tier interference, the signal transmission of the small cells must not cause interference to the MUEs. From (2), the constraints of zero cross-tier interference to $\mathrm{UE}_{0, \pi(0)}$ are imposed by

$$
\boldsymbol{U}_{0, \pi(0)}^{H} \boldsymbol{H}_{0, \pi(0), \ell} \boldsymbol{V}_{\ell, \pi(\ell)}=\mathbf{0}, \quad \ell \in \mathcal{L} .
$$

The elimination of cross-tier interference leads to the received signal at $\mathrm{UE}_{0, \pi(0)}$ given by

$$
\boldsymbol{r}_{0, \pi(0)}=U_{0, \pi(0)}^{H} H_{0, \pi(0), 0} V_{0, \pi(0)} x_{0, \pi(0)}+U_{0, \pi(0)}^{H} z_{0, \pi(0)}
$$

Then, the precoding matrix $\boldsymbol{V}_{0, \pi(0)}$ at the MBS and the postprocessing matrix $\boldsymbol{U}_{0, \pi(0)}$ at the MUE are designed to maximize its rate. From (6), the link between the MBS and MUE is equivalent to a MIMO single user channel and, therefore, the MBS can apply eigenmode transmission [27]. The singular value decomposition (SVD) of channel matrix $\boldsymbol{H}_{0, \pi(0), 0}$ is given by

$$
\boldsymbol{H}_{0, \pi(0), 0}=\Psi_{0, \pi(0)} \boldsymbol{\Sigma}_{0, \pi(0)} \mathbf{\Upsilon}_{0, \pi(0)}^{H}
$$

where $\boldsymbol{\Psi}_{0, \pi(0)}$ and $\boldsymbol{\Upsilon}_{0, \pi(0)}$ are respectively the left and right singular vector matrices of $\boldsymbol{H}_{0, \pi(0), 0}$ while $\boldsymbol{\Sigma}_{0, \pi(0)}$ is a diagonal matrix whose diagonal elements are the decreasing ordered singular values. Accordingly, the precoding matrix at the MBS and the postprocessing matrix at the MUE are respectively chosen by

$$
\begin{gathered}
\boldsymbol{V}_{0, \pi(0)}=\operatorname{col}_{1}^{d_{0, \pi(0)}}\left(\boldsymbol{\Upsilon}_{0, \pi(0)}\right) \in C^{N_{0} \times d_{0, \pi(0)}} \\
\boldsymbol{U}_{0, \pi(0)}=\operatorname{col}_{1}^{d_{0, \pi(0)}}\left(\boldsymbol{\Psi}_{0, \pi(0)}\right) \in C^{M_{0, \pi(0)} \times d_{0, \pi(0)}}
\end{gathered}
$$

The optimal covariance matrix $\boldsymbol{Q}_{0, \pi(0)}$ must be diagonal, i.e., $\boldsymbol{Q}_{0, \pi(0)}=\operatorname{diag}\left(q_{0, \pi(0), 1}, q_{0, \pi(0), 2}, \ldots, q_{0, \pi(0), d_{0, \pi(0)}}\right)$ where $q_{0, \pi(0), i} \geq 0$ denotes the transmit power allocated to the $i$-th data stream at $\mathrm{UE}_{0, \pi(0)}[27]$. Then, the achievable rate of the MBS link from (6) is computed by

$$
\mathcal{R}_{0, \pi(0)}=\sum_{i=1}^{d_{0, \pi(0)}} \log _{2}\left(1+\frac{q_{0, \pi(0), i} \Sigma_{0, \pi(0)}^{2}(i, i)}{\sigma_{0, \pi(0)}^{2}}\right) .
$$

The optimally allocated power is found to maximize the channel capacity as follows

$$
\begin{array}{rc}
\max _{\boldsymbol{Q}_{0, \pi(0)} \geq 0} & \sum_{i=1}^{d_{0, \pi(0)}} \log _{2}\left(1+\frac{q_{0, \pi(0), i} \Sigma_{0, \pi(0)}^{2}(i, i)}{\sigma_{0, \pi(0)}^{2}}\right) \\
\text { s.t. } & \sum_{i=1}^{d_{0, \pi(0)}} q_{0, \pi(0), i} \leq P_{0}^{\max }
\end{array}
$$

where $P_{0}^{\max }$ is the maximum transmit power at the MBS. The optimal power allocation to problem (11) can be found by the water-filling algorithm given by [27]

$$
q_{0, \pi(0), i}=\left[\mu-\frac{\sigma_{0, \pi(0)}^{2}}{\Sigma_{0, \pi(0)}^{2}(i, i)}\right]^{+}
$$

where the water level $\mu$ is chosen to satisfy the power constraint (11b). The design steps of the macrocell transmission are summarized in Algorithm 1.

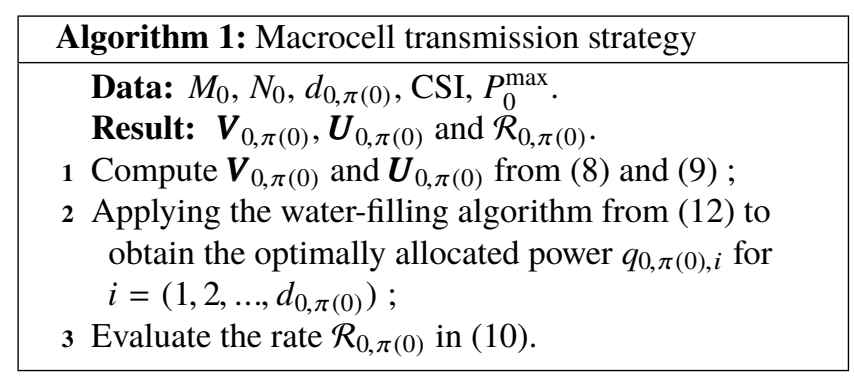

Note that the MBS only uses its local CSI from the MBS to the MUE in order to maximize its rate. Therefore, there is no requirement of CSI exchange from the SBSs to the MBS.

\subsection{Small Cell Transmission Strategies}

As mentioned earlier that the SBSs can operate in the frequency owned by the MBS if they do not cause harmful interference to the MUE [1], [19]. This means that the SBSs should deal with cross-tier interference to the MUE. To completely cancel interference to $\mathrm{UE}_{0, \pi(0)}$ in the macrocell, we design the precoder matrices of the SBSs as the cascade of two precoding matrices

$$
\boldsymbol{V}_{\ell, k}=\boldsymbol{A}_{\ell, k} \boldsymbol{B}_{\ell, k} .
$$

Here, $\boldsymbol{A}_{\ell, k} \in C^{N_{\ell} \times N_{\ell}-d_{0, \pi(0)}}$ is used to suppress cross-tier interference to the MUE. From (5), matrix $\boldsymbol{A}_{\ell, k}$ is chosen as

$$
\boldsymbol{A}_{\ell, k}=\mathcal{N}\left[\boldsymbol{U}_{0, \pi(0)}^{H} \boldsymbol{H}_{0, \pi(0), \ell}\right]
$$

where $\mathcal{N}[\boldsymbol{X}]$ is an orthogonal basis for the null space of $\boldsymbol{X}$. Note that in order to find $\boldsymbol{A}_{\ell, k}$ the SBSs use their local CSI, i.e., CSI associated with the SBSs. In addition, in HetNets, the MBS and SBS can share CSI via a low-latency backhaul [4], [6]. Thus, information $\boldsymbol{U}_{0, \pi(0)}$ is assumed to be available at the SBSs. Additionally, the SBSs can generate the random matrices $\boldsymbol{B}_{\ell, k} \in C^{N_{\ell}-d_{0, \pi(0)} \times d_{\ell, k}}$ and broadcast them to their users. Alternatively, the pre-defined pseudorandom matrices can be used and are known to all users in the network [9], [16]. On the other hand, to mitigate co-tier interference at the SUEs, the $\ell$-th SBS will opportunistically select a user among its associated $F_{\ell}$ users to communicate with. To reduce overhead exchange information between the SBSs and SUEs, we assume that only local CSI is available at the SUEs. In addition, during the user selection, equal power allocation (EPA) to data streams is assumed, i.e.,

$$
\boldsymbol{Q}_{\ell, \pi(\ell)}=\frac{P_{\ell}^{\max }}{d_{\ell, \pi(\ell)}} \boldsymbol{I}_{d_{\ell, \pi(\ell)}}, \ell \in \mathcal{L} .
$$

In what follows, we present two scheduling schemes. 


\subsubsection{Minimum Interference Leakage Based User Selec- tion (MILUS)}

In the MILUS scheme, the SUE receiver in which interference from other cells can be most aligned will be selected. From (2), interference leakage from the $i$-th BS to user $k$ in cell $\ell$ with $(i \neq \ell)$ is given by

$$
\boldsymbol{\Gamma}_{\ell, k, i}\left(\boldsymbol{U}_{\ell, k}\right)=\left\langle\frac{P_{i}^{\max }}{d_{i, \pi(i)}} \boldsymbol{U}_{\ell, k}^{H} \boldsymbol{H}_{\ell, k, i} \boldsymbol{V}_{i, \pi(i)} \boldsymbol{V}_{i, \pi(i)}^{H} \boldsymbol{H}_{\ell, k, i}^{H} \boldsymbol{U}_{\ell, k}\right\rangle .
$$

Accordingly, the total of interference leakage at the $k$-th user in cell $\ell$ is

$$
\boldsymbol{\Gamma}_{\ell, k}\left(\boldsymbol{U}_{\ell, k}\right)=\sum_{i=0, i \neq \ell}^{L} \boldsymbol{\Gamma}_{\ell, k, i}\left(\boldsymbol{U}_{\ell, k}\right)=\left\langle\boldsymbol{U}_{\ell, k}^{H} \boldsymbol{\Psi}_{\ell, k} \boldsymbol{U}_{\ell, k}\right\rangle
$$

where $\boldsymbol{\Psi}_{\ell, k}=\sum_{i=0, i \neq \ell}^{L} \frac{P_{i}^{\max }}{d_{i, \pi(i)}} \boldsymbol{H}_{\ell, k, i} \boldsymbol{V}_{i, \pi(i)} \boldsymbol{V}_{i, \pi(i)}^{H} \boldsymbol{H}_{\ell, k, i}^{H}$ is the interference covariance matrix at the $k$-th user in the $\ell$-th small cell. As shown in [28], each user can estimate the associated interference covariance matrix. The minimum interference leakage is used as a metric to select a user. Then, each SUE computes the minimum interference leakage by solving the following problem:

$$
\min _{\boldsymbol{U}_{\ell, k}^{H} \boldsymbol{U}_{\ell, k}=\boldsymbol{I}_{d_{\ell, k}}} \boldsymbol{\Gamma}_{\ell, k}\left(\boldsymbol{U}_{\ell, k}\right)
$$

which results in $\boldsymbol{U}_{\ell, k}=v_{\min }^{d_{\ell, k}}\left(\boldsymbol{\Psi}_{\ell, k}\right)$, where $v_{\min }^{d_{\ell, k}}(\boldsymbol{X})$ is a matrix whose columns are the $d_{\ell, k}$ eigenvectors corresponding to the $d_{\ell, k}$ smallest eigenvalues of $\boldsymbol{X}$ [29]. Note that to calculate the interference leakage $\Gamma_{\ell, k}$, the SUE receiver only requires local channel information, i.e., the channels from the BSs to its receiver. Then, it reports this metric to its associated SBS. The $\ell$-th SBS will choose the $\pi(\ell)$-th user which has the smallest interference leakage, i.e.,

$$
\pi(\ell)=\arg \min _{k \in \mathcal{F}_{\ell}} \Gamma_{\ell, k}, \quad \ell \in \mathcal{L} .
$$

\subsubsection{Maximum SNR Based User Selection (MSNRUS)}

In contrast to the MILUS method, in the MSNRUS scheme the SUE will be selected by using the maximum signal to noise ratio (SNR) criteria. In light of (2), the SNR at receiver $k$ in cell $\ell$ is defined by

$$
\gamma_{\ell, k}\left(\boldsymbol{U}_{\ell, k}\right)=\frac{\left\langle\boldsymbol{U}_{\ell, k}^{H} \boldsymbol{\Omega}_{\ell, k} \boldsymbol{U}_{\ell, k}\right\rangle}{\left\langle\sigma_{\ell, k}^{2} \boldsymbol{U}_{\ell, k}^{H} \boldsymbol{U}_{\ell, k}\right\rangle}, \quad \ell \in \mathcal{L}, \text { and } k \in \mathcal{F}_{\ell}
$$

where $\boldsymbol{\Omega}_{\ell, k}=\frac{P_{\ell}^{\max }}{d_{\ell, \pi(\ell)}} \boldsymbol{H}_{\ell, k, \ell} \boldsymbol{V}_{\ell, k} \boldsymbol{V}_{\ell, k}^{H} \boldsymbol{H}_{\ell, k, \ell}^{H}$ is the covariance matrix of the desired signal at the $k$-th user in the $\ell$-th small cell. The UE receiver calculates its maximum SNR by

$$
\max _{\boldsymbol{U}_{\ell, k}^{H} \boldsymbol{U}_{\ell, k}=\boldsymbol{I}_{d_{\ell, k}}} \gamma_{\ell, k}\left(\boldsymbol{U}_{\ell, k}\right)
$$

which results in $\boldsymbol{U}_{\ell, k}=v_{\max }^{d_{\ell, k}}\left(\boldsymbol{\Omega}_{\ell, k}\right)$ where $v_{\max }^{d_{\ell, k}}(\boldsymbol{X})$ is a matrix whose columns are the $d_{\ell, k}$ eigenvectors corresponding to the $d_{\ell_{k}}$ largest eigenvalues of $\boldsymbol{X}$ [29]. Each SUE feeds its SNR $\gamma_{\ell, k}$ back its corresponding SBS and, then, the SBS will select the SUE with the highest SNR, i.e.

$$
\pi(\ell)=\arg \max _{k \in \mathcal{F}_{\ell}} \gamma_{\ell, k} \quad \ell \in \mathcal{L} .
$$

\subsection{Optimal Power Allocation Scheme at SBSs}

It should be noted that since it is difficult for all UEs to obtain global CSI of all links, the above user selection schemes only exploit local CSI associated with each UE. In practice, the SBSs can be connected to a central unit (CU) via a backhaul network, and, thus, the SBSs can exchange CSI of the selected users associated to them [1], [4]. Given CSI of the selected links, the SBSs can cooperate to jointly optimize the power allocation in order to improve the sum rate of the small cells ${ }^{2}$. The problem of interest is to find the transmitted signal covariance matrices at the SBSs to maximize the total sum rate of $L$ small cells. Thus, the optimal design of transmitted signal covariance matrices can be written as

$$
\begin{array}{cl}
\max _{\boldsymbol{Q}} & \mathcal{R}(\boldsymbol{Q})=\sum_{\ell=1}^{L} \mathcal{R}_{\ell, \pi(\ell)}(\boldsymbol{Q}) \\
\text { s.t. } & \left\langle\boldsymbol{Q}_{\ell, \pi(\ell)}\right\rangle \leq P_{\ell}^{\max }, \ell \in \mathcal{L}
\end{array}
$$

where we have defined $\boldsymbol{Q}=\left\{\boldsymbol{Q}_{\ell, \pi(\ell)}\right\}_{\ell=1}^{L}$ for simplicity of notation. Constraints (23b) are imposed on the transmit power per SBS where $P_{\ell}^{\max }$ is the maximum transmit power at SBS $\ell$. It can be verified that the power constraints of (23b) are convex while the objective function (23a) is nonconcave. Thus, problem (23) is nonconvex which renders the mathematical challenges to find the optimal solutions of (23). As shown in [25], [30], the rate expression (3) can be naturally presented as a difference of two convex functions. Thus, we aim to reformulate the optimization problem (23) as d.c. optimization and, then, develop an iterative d.c. programming to find the transmit signal covariance matrices. To this end, we decompose the rate in (23a) as

$\mathcal{R}_{\ell, \pi(\ell)}(\boldsymbol{Q})=-\log _{2}\left|\boldsymbol{R}_{\ell, \pi(\ell)}\right|+\log _{2}\left|\boldsymbol{R}_{\ell, \pi(\ell)}+\tilde{\boldsymbol{H}}_{\ell, \ell} \boldsymbol{Q}_{\ell, \pi(\ell)} \tilde{\boldsymbol{H}}_{\ell, \ell}^{H}\right|$

where $\tilde{\boldsymbol{H}}_{\ell, i}=\boldsymbol{U}_{\ell, \pi(\ell)}^{H} \boldsymbol{H}_{\ell, \pi(\ell), i} \boldsymbol{V}_{i, \pi(i)}$ and

$$
\boldsymbol{R}_{\ell, \pi(\ell)}=\sigma_{\ell, \pi(\ell)}^{2} \boldsymbol{I}_{d_{\ell, \pi(\ell)}}+\sum_{i=0, i \neq \ell}^{L} \tilde{\boldsymbol{H}}_{\ell, i} \boldsymbol{Q}_{i, \pi(i)} \tilde{\boldsymbol{H}}_{\ell, i}^{H} .
$$

For simplicity of exposition, we define

$$
\begin{gathered}
f_{\ell}(\boldsymbol{Q})=-\log _{2}\left|\boldsymbol{R}_{\ell, \pi(\ell)}\right| \\
g_{\ell}(\boldsymbol{Q})=-\log _{2}\left|\boldsymbol{R}_{\ell, \pi(\ell)}+\tilde{\boldsymbol{H}}_{\ell, \ell} \boldsymbol{Q}_{\ell, \pi(\ell)} \tilde{\boldsymbol{H}}_{\ell, \ell}^{H}\right| .
\end{gathered}
$$

${ }^{2}$ It should be noted that the sum-rate of the network can be improved if the user selection and power allocation is jointly designed. However, such a design problem is intractable and requires the global CSI of all users in the network resulting in high demanding information exchange. 
It is obvious that $f_{\ell}(\boldsymbol{Q})$ and $g_{\ell}(\boldsymbol{Q})$ are convex functions [32]. In light of equations (24), (25) and (26), the sum rate $\mathcal{R}(\boldsymbol{Q})$ in (23a) can be rewritten as

$$
\mathcal{R}(\boldsymbol{Q})=-[g(\boldsymbol{Q})-f(\boldsymbol{Q})]
$$

where $g(\boldsymbol{Q})=\sum_{\ell=1}^{L} g_{\ell}(\boldsymbol{Q})$ and $f(\boldsymbol{Q})=\sum_{\ell=1}^{L} f_{\ell}(\boldsymbol{Q})$ is a positive sum of convex functions and, therefore, they are also convex [32]. Then, the optimization problem (23) can be equivalently transformed into

$$
\begin{aligned}
& \min _{\boldsymbol{Q}} g(\boldsymbol{Q})-f(\boldsymbol{Q}) \\
& \text { s.t. }\left\langle\boldsymbol{Q}_{\ell, \pi(\ell)}\right\rangle \leq P_{\ell}^{\max }, \ell \in \mathcal{L} .
\end{aligned}
$$

That is, the optimization problem (28) is in form of d.c. programming. The basic idea to tackle d.c. optimization is to convexify $f(\boldsymbol{Q})$ to obtain the convex objective function. We can approximate the nonconvex part of the objective function by using its convex majorant [25], [30], [31]. Given $\boldsymbol{Q}^{(\kappa)}$ at the $\kappa$-th iteration, one has

$$
f(\boldsymbol{Q}) \approx f\left(\boldsymbol{Q}^{(\kappa)}\right)+\sum_{\ell=1}^{L} \sum_{i=1, i \neq \ell}^{L}\left\langle\boldsymbol{\Pi}_{\ell, i}, \boldsymbol{Q}_{i, \pi(i)}-\boldsymbol{Q}_{i, \pi(i)}^{(\kappa)}\right\rangle
$$

where $\boldsymbol{\Pi}_{\ell, i}$ is the partial derivative of $f_{\ell}(\boldsymbol{Q})$ with respect to $\boldsymbol{Q}_{i, \pi(i)}$ evaluated at $\boldsymbol{Q}^{(\kappa)}$, defined by

$$
\boldsymbol{\Pi}_{\ell, i}=\left.\frac{\partial f_{\ell}(\boldsymbol{Q})}{\partial \boldsymbol{Q}_{i_{\pi(i)}}}\right|_{\boldsymbol{Q}=\boldsymbol{Q}^{(\kappa)}}=\tilde{\boldsymbol{H}}_{\ell, i}^{H}\left(\boldsymbol{R}_{\ell, \pi(\ell)}^{(\kappa)}\right)^{-1} \tilde{\boldsymbol{H}}_{\ell, i}
$$

Accordingly, the optimization problem (28) can be recast as

$$
\begin{aligned}
& \min _{\boldsymbol{Q}} g(\boldsymbol{Q})-f\left(\boldsymbol{Q}^{(\kappa)}\right)-\sum_{\ell=1}^{L} \sum_{i=1, i \neq \ell}^{L}\left\langle\boldsymbol{\Pi}_{\ell, i}, \boldsymbol{Q}_{i, \pi(i)}-\boldsymbol{Q}_{i, \pi(i)}^{(\kappa)}\right\rangle \\
& \text { s.t. }\left\langle\boldsymbol{Q}_{\ell, \pi(\ell)}\right\rangle \leq P_{\ell}^{\max }, \ell \in \mathcal{L} .
\end{aligned}
$$

It is obvious that problem (31) is convex optimization since it minimizes the convex objective function over the convex constraints. It is well-known that convex optimization problem can be efficiently solved by available convex programming solvers. As a result, to obtain the optimal solution to the d.c. programming (28), we solve a sequence of the convex optimization problem (31). In summary, the iterative procedure to solve problem (23) is described in Algorithm 2 where $\epsilon$ is an acceptable accuracy.

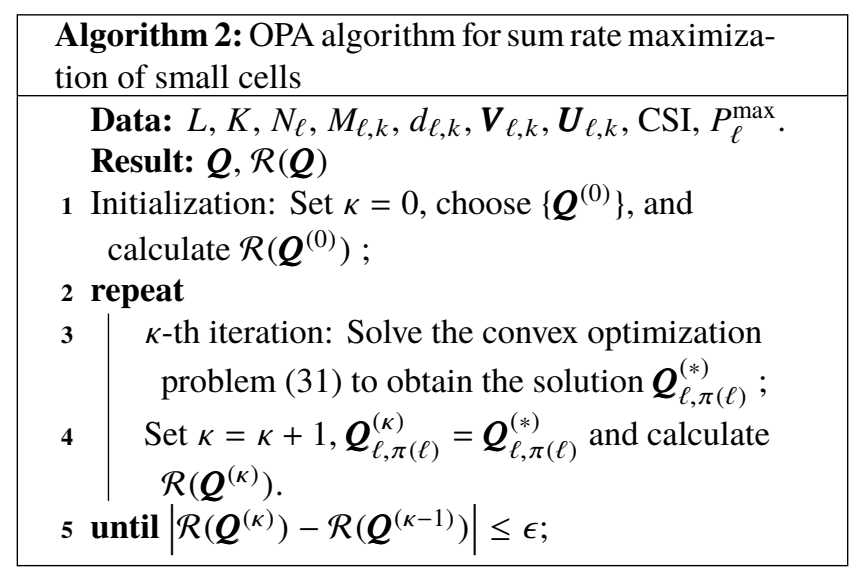

Since the original optimization problem (23) is nonconvex, it is important to show the convergence of the iterative algorithm. Since $\boldsymbol{f}_{\ell}(Q)$ is convex, it implies that [32]

$$
f(\boldsymbol{Q}) \geq f\left(\boldsymbol{Q}^{(\kappa)}\right)+\sum_{\ell=1}^{L} \sum_{i=1, i \neq \ell}^{L}\left\langle\boldsymbol{\Pi}_{\ell, i}, \boldsymbol{Q}_{i, \pi(i)}-\boldsymbol{Q}_{i, \pi(i)}^{(\kappa)}\right\rangle .
$$

Denote the objective function of problem $(31)$ by $-\tilde{\mathcal{R}}(\boldsymbol{Q})$. Then, we have $\mathcal{R}(\boldsymbol{Q}) \geq \tilde{\mathcal{R}}(\boldsymbol{Q}), \forall \boldsymbol{Q}$, which results in

$$
\mathcal{R}\left(\boldsymbol{Q}^{(\kappa+1)}\right) \geq \tilde{\mathcal{R}}\left(\boldsymbol{Q}^{(\kappa+1)}\right) .
$$

On the other hand, at the $\kappa$-th iteration in which $Q^{(\kappa+1)}$ is an optimal solution to (31), one has

$$
\tilde{\mathcal{R}}\left(\boldsymbol{Q}^{(\kappa+1)}\right) \geq \tilde{\mathcal{R}}\left(\boldsymbol{Q}^{(\kappa)}\right)=\mathcal{R}\left(\boldsymbol{Q}^{(\kappa)}\right) .
$$

Combining (33) and (34) yields $\mathcal{R}\left(\boldsymbol{Q}^{(\kappa+1)}\right) \geq \mathcal{R}\left(\boldsymbol{Q}^{(\kappa)}\right)$. This means that the total sum rate of the small cells is monotonically nondecreasing over iteration. Furthermore, given the power constraints, the system sum rate is upper bounded. Thus, the convergence of the d.c. programming in Algorithm 2 is guaranteed.

\section{Illustrative Results}

In this section, we provide numerical simulation results to verify the effectiveness of the proposed method in terms of the sum rate. The simulation parameters are shown in Tab. 1 [23]. There are two small cells deployed in the hotspot area of the macrocell. The small cells are uniformly distributed in the circle of the radius $333 \mathrm{~m}$ from the MBS. The channel coefficients are generated as the products of two components: the first components account for the path loss and shadowing effect given in Tab. 1 and the second components represent the Rayleigh small-scale fading generated from i.i.d. complex Gaussian distribution variables with $C \mathcal{N}(0,1)$. All results are averaged over user locations and channel realizations. Consider that the MBS and SBSs are equipped with 8 and 4 antennas, respectively while each UE has 2 antennas. Each BS transmits one data stream to its associated users. The acceptable accuracy for the iterative algorithm is set $\epsilon=10^{-9}$.

\begin{tabular}{c|c}
\hline Parameter & Value \\
\hline \hline Macro cell radius & $500 \mathrm{~m}$ \\
\hline Small cell radius & $40 \mathrm{~m}$ \\
\hline Path loss from MBS to UE & $128.1+37.6 \log _{10}(d) d$ in km \\
\hline Path loss from SBS to UE & $140.7 .1+36.7 \log _{10}(d) d$ in km \\
\hline System bandwidth & $10 \mathrm{MHz}$ \\
\hline Noise power density & $-174 \mathrm{dBm} / \mathrm{Hz}$ \\
\hline Noise figure & $9 \mathrm{~dB}$ \\
\hline Shadowing standard deviation & $8 \mathrm{~dB}$ \\
\hline
\end{tabular}

Tab. 1. Simulation parameters. 
First, we examine the sum rate performance of the proposed schemes with MILUS and MSNRUS in the cases of EPA and OPA. We set $L=2, F_{0}=4, F_{\ell}=2, \ell \in \mathcal{L}$ and $P_{\ell}^{\max }=P_{\max }=0.5 P_{0}^{\max }$. The average sum-rates are shown in Fig. 2. It is worth noting that both user selection schemes guarantee zero cross-tier interference to the MUE. Thus, the rate of the macrocell increases with the transmit power. It can be seen from Fig. 2 that as compared with the EPA methods, the OPA schemes can improve the sum rate of the small cells for both user selection schemes With regard to user selection strategies, as observed from Fig. 2 that the MSNRUS outperforms the MILUS at the low transmit power region while the former is inferior to the latter at the high transmit power. The reason is that the MILUS focuses on mitigating interference while MSNRUS aims at maximizing SNR. At high SNR the system is interference limited, and, thus, the sum-rate of the MSNRUS is lower than that of the MILUS.

Now, we study on the achievable sum rate of the system for different number of UEs per small cell. We consider $\{1,2,4\}$ users per small cell. Figures 3 and 4 respectively show the sum rate of the small cells for the MILUS and MSNRUS with different number of users. For the sake of comparison, the sum rate performance of the transmission strategies with EPA is also shown. We can see that when

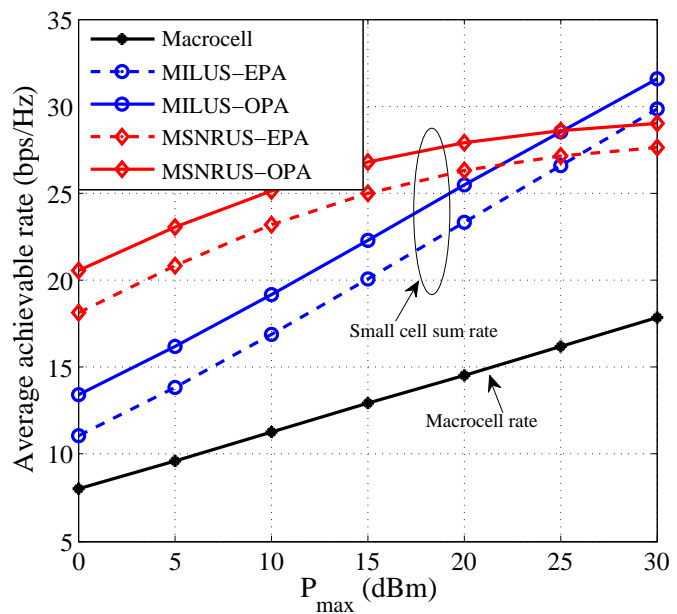

Fig. 2. Average achievable rate of the proposed method.

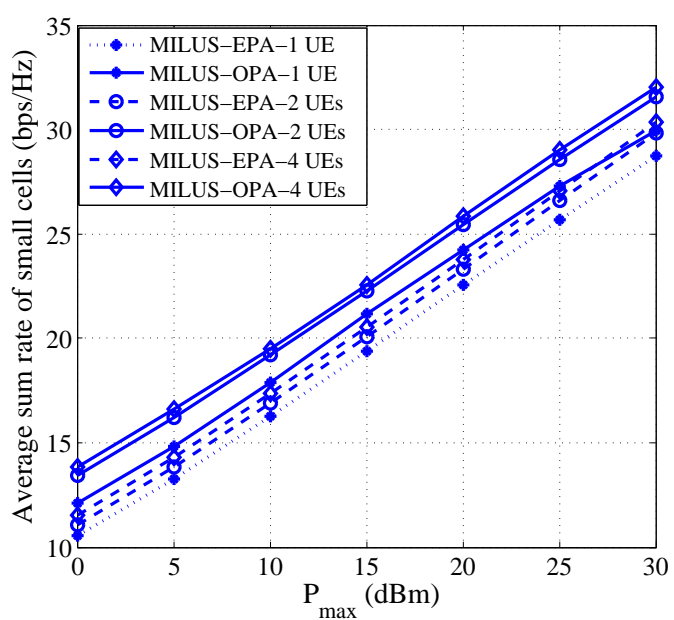

Fig. 3. Average achieved sum rate of the small cells for MLIUS. the number of users increases the sum rate is improved. The reason is that it is more likely to select the best user among the higher number of users. In addition, the user scheduling schemes with OPA outperform those of EPA. It reveals that the optimal power allocation is of importance in interference channels.

We compare the sum rate performance of our proposed method with two baselines, namely the TDMA scheme [8], [23] and the selfish approach [33]. In the TDMA scheme, each cell transmission is accomplished into different time slots and, thus, there is no inter-cell interference [8], [23]. Each BS will select its severing user by using the channel strength (cf. (4)), and exploit the water-filling power allocation to eigmode transmission. In the selfish approach, each BS only considers maximizing its own data rate [33]. Thus, in the selfish approach, the BS will use the MSNRUS to select the user and apply the water-filling algorithm to maximize its own data rate. For fair comparison, we compare these two baselines with our proposed MSNRUS-OPA method. As can be observed from Fig. 5, our proposed method and the selfish approach outperform the TDMA scheme. However, the selfish approach provides a lower rate of the macrocell than our proposed method. This is because the selfish approach

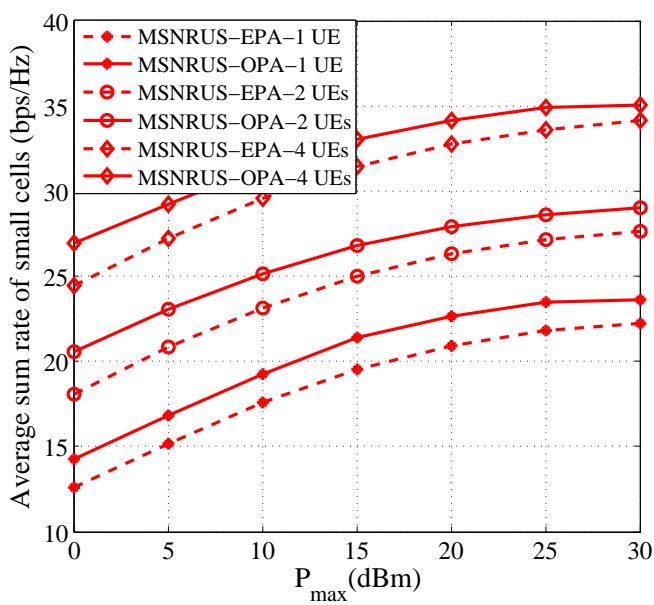

Fig. 4. Average achieved sum rate of the small cells for MSNRUS.

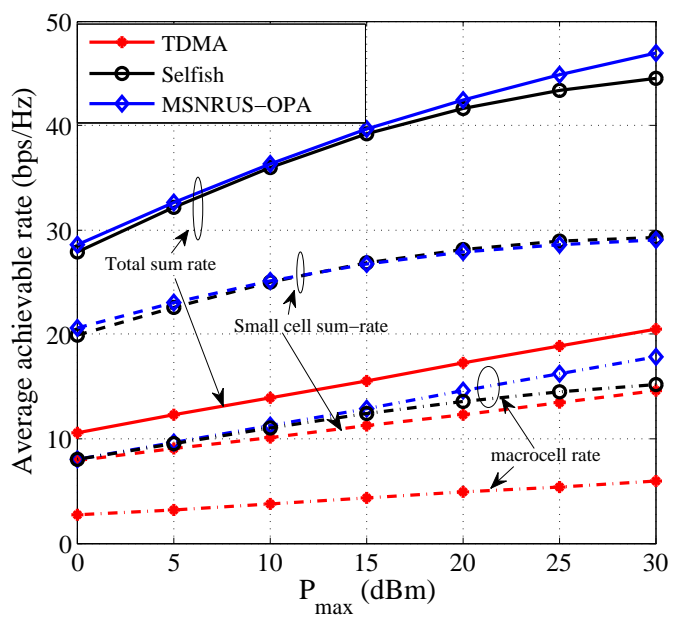

Fig. 5. Average sum rate comparison between our method with the TDMA and selfish approaches. 
does not handle interference to the MUE while our proposed method guarantees zero-interference to the MUE. Accordingly, the total sum rate of our performance is superior to that of the selfish approach.

\section{Conclusion}

This paper has presented the transmission strategies for the downlink of multicell multiuser MIMO HetNets. The transmission strategy of the macrocell is to exploit the eigenmode transmission while the small cells opportunistically select their corresponding users subject to no cross-tier interference to the MUE. To handle the cross-tier interference, we have imposed the structure on the precoders at the SBSs. We have also investigated two OIA schemes by using the minimum interference leakage and maximum SNR scheduling metrics. In addition, given the limited coordination of the BSs, we have also developed the d.c. iterative algorithm for OPA. The simulation results are shown that the proposed approaches outperform the TDMA and selfish methods in terms of the sum rate and that the OPA algorithms can improve the sum rate as compared to the EPA schemes.

\section{Acknowledgments}

This research is funded by Vietnam National Foundation for Science and Technology Development (NAFOSTED) under grant number 102.04-2013.46.

\section{References}

[1] CASTANHEIRA, D., SILVA, A., GAMEIRO, A. Null-space cognitive precoding for heterogeneous networks. IET Communications, 2014, vol. 8, no. 5, p. 555-563. DOI: 10.1049/iet-com.2013.043

[2] MANOSHA, K. B. S., CODREANU, M., RAJATHEVA, N., et al. Power-throughput tradeoff in MIMO heterogeneous networks. IEEE Transactions on Wireless Communications, 2014, vol. 13, no. 8, p. 4309-4322. DOI: 10.1109/TWC.2014.2319076

[3] DAMnjanOVIC, A., MONTOJO, J., WEI, Y., et al., A survey on 3GPP heterogeneous networks. IEEE Wireless Communications, 2011, vol. 18, no. 3, p. 10-21. DOI: 10.1109/MWC.2011.5876496

[4] XU, Z., YANG, C., LI, G. Y., et al. Energy-efficient CoMP precoding in heterogeneous networks. IEEE Transactions on Signal Processing, 2014, vol. 62, no. 4, p. 1005-1017. DOI: 10.1109/TSP.2013.2296279

[5] SHIN, W., LEE, N., NOH, W., et al. Hierarchical interference alignment for heterogeneous networks with multiple antennas. In Proceedings of the IEEE International Conference on Communications Workshops (ICC). Kyoto (Japan), 2011, p. 1-6. DOI: $10.1109 /$ iccw.2011.5963547

[6] RIHAN, M., ELSABROUTY, M., MUTA, O., et al. Iterative interference alignment in macrocell-femtocell networks: A cognitive radio approach. In Proceedings of the 11th International Symposium on Wireless Communications Systems (ISWCS). Barcelona (Spain), 2014, p. 654-658. DOI: 10.1109/ISWCS.2014.6933435
[7] AMIR, M., EL-KEYI, A., NAFIE, M. Constrained interference alignment and the spatial degrees of freedom of MIMO cognitive networks. IEEE Transactions on Information Theory, 2011, vol. 57, no. 5, p. 2994-3004. DOI: 10.1109/TIT.2011.2119770

[8] SHIN, W., NOH, W., JANG, K., et al. Herarchical interference alignment for downlink heterogeneous networks. IEEE Transactions on Wireless Communications, 2012, vol. 11, no. 12, p. 4549-4559. DOI: 10.1109/TWC.2012.101912.120421

[9] JUNG, B. C., PARK, D., SHIN, W. Y. Opportunistic interference mitigation achieves optimal degrees-of-freedom in wireless multi-cell uplink networks. IEEE Transactions on Communications, 2012, vol. 60, no. 7, p. 1935-1944. DOI: 10.1109/TCOMM.2012.051012.110205

[10] CADAMBE, V. R., JAFAR, S. A. Interference alignment and degrees of freedom of the K-user interference channel. IEEE Transactions on Information Theory, 2008, vol. 54, no. 8, p. 3425-3441. DOI: 10.1109/TIT.2008.926344

[11] VU, T. T., KHA, H. H., DUONG, T. Q. Interference alignment designs for secure multiuser MIMO systems: Rank constrained rank minimization approach. In Proceedings of the International Conference on Communications, Management and Telecommunications (ComManTel). DaNang (Vietnam), 2015, p. 116-121. DOI: 10.1109/ComManTel.2015.7394271

[12] VU, T. T., KHA, H. H., DUONG, T. Q., et al. Wiretapped signal leakage minimization for secure multiuser MIMO systems via interference alignment. In Proceedings of the International Conference on Advanced Technologies for Communications (ATC). Ho Chi Minh City (Vietnam), 2015, p. 79-83. DOI: 10.1109/ATC.2015.7388422

[13] VU, T. T., KHA, H. H., TUAN, H. D. Transceiver design for optimizing the energy efficiency in multiuser MIMO channels. IEEE Communications Letters, 2016, vol. 20, no. 8, p. 1507-1510. DOI: 10.1109/LCOMM.2016.2579640

[14] Ali, S. S., CASTANHEIRA, D., SIlva, A., et al. Transmission cooperative strategies for MIMO-OFDM heterogeneous networks. Radioengineering, 2015, vol. 24, no. 2, p. 431-441. DOI: $10.13164 /$ re.2015.0431

[15] SHU, C., HO, M., TSE, D. N. C. Downlink interference alignment. IEEE Transactions on Communications, 2011, vol. 59, no. 9, p. 2616-2626. DOI: 10.1109/TCOMM.2011.070511.100313

[16] JIN, H., JEON, S. W., JUNG, B. C. Opportunistic iterference alignment for random access networks. IEEE Transactions on Vehicular Technology, 2015, vol. 64, no. 12, p. 5947-5954. DOI: 10.1109/TVT.2015.2388714

[17] ZHAO, N., YU, F. R., LEUNG, V. C. M. Opportunistic communications in interference alignment networks with wireless power transfer. IEEE Wireless Communications, 2015, vol. 22, no. 1, p. 88-95. DOI: 10.1109/MWC.2015.7054723

[18] TANG, J., ANANDKUMAR, A. J. G., LAMBOTHARAN, S. Opportunistic MIMO multi-cell interference alignment techniques. In Proceedings of the 5th International Conference on Internet Multimedia Systems Architecture and Application (IMSAA). Bangalore, 2011, p. 1-4. DOI: 10.1109/IMSAA.2011.6156355

[19] RIHAN, M., ELSABROUTY, M., MUTA, O., et al. Interference mitigation framework based on interference alignment for femtocellmacrocell two tier cellular systems. IEICE Transactions on Communication, 2015, vol. E98-B, no. 3, p. 467-476. ISSN: 1745-1345

[20] LEE, J. H., CHOI, W. Interference alignment by opportunistic user selection in 3-user MIMO interference channels. In Proceddings of the IEEE International Conference on Communications (ICC). Kyoto (Japan), 2011, p. 1-5. DOI: 10.1109/icc.2011.5962830 
[21] KANG, X., LIANG, Y. C., GARG, H. K. Distributed power control for spectrum-sharing femtocell networks using stackelberg game. In Proceedings of the IEEE International Conference on Communications (ICC). Kyoto (Japan), 2011, p. 1-5. DOI: 10.1109/icc.2011.5962650

[22] OH, D. C., LEE, H. C., LEE, Y. H. Power control and beamforming for femtocells in the presence of channel uncertainty. IEEE Transactions on Vehicular Technology, 2011, vol. 60, no. 6, p. 2545-2554. DOI: 10.1109/TVT.2011.2158615

[23] LIU, G., SHENG, M., WANG, X., et al. Interference alignment for partially connected downlink MIMO heterogeneous networks. IEEE Transactions on Communications, 2015, vol. 63, no. 2, p. 551-564. DOI: 10.1109/TCOMM.2015.2388450

[24] PANTISANO, F., BENNIS, M., SAAD, W., et al. Interference alignment for cooperative femtocell networks: A game-theoretic approach IEEE Transactions on Mobile Computing, 2013, vol. 12, no. 11, p. 2233-2246. DOI: 10.1109/TMC.2012.196

[25] KHA, H. H., TUAN, H. D., NGUYEN, H. H. Fast gobal optimal power allocation in wireless networks by local D.C. programming. IEEE Transactions on Wireless Communications, 2012, vol. 11, no. 2, p. 510-515. DOI: 10.1109/TWC.2011.120911.110139

[26] ZOU, Y., LI, X., LIANG, Y. C. Secrecy outage and diversity analysis of cognitive radio systems. IEEE Journal on Selected Areas in Communications, 2014, vol. 32, no. 11, p. 2222-2236. DOI: 10.1109/JSAC.2014.141121

[27] PALOMAR, D. P., FONOLlOSA, J. R. Practical algorithms for a family of waterfilling solutions. IEEE Transactions on Signal Processing, 2005, vol. 53, no. 2, p. 686-695. DOI: $10.1109 / \mathrm{TSP} .2004 .840816$

[28] SHIM, S., KWAK, J. S., HEATH, R. W., et al. Block diagonalization for multi-user MIMO with other-cell interference. IEEE Transactions on Wireless Communications, 2008, vol. 7, no. 7, p. 2671-2681. DOI: 10.1109/TWC.2008.070093

[29] HORN, R. A., JOHNSON, C. R. Matrix Analysis. New York (USA): Cambridge University Press, 2012. ISBN: 0521548233

[30] RASHID, U., TUAN, H. D., KHA, H. H., et al. Joint optimization of source precoding and relay beamforming in wireless MIMO relay networks. IEEE Transactions on Communications, 2014, vol. 62 , no. 2, p. 488-499. DOI: 10.1109/TCOMM.2013.122013.130239
[31] NGUYEN, D. H. N., NGUYEN-LE, H., LE-NGOC, T. Blockdiagonalization precoding in a multiuser multicell MIMO system: Competition and coordination. IEEE Transactions on Wireless Communications, 2014, vol. 13, no. 2, p. 968-981. ISSN: 1536-1276. DOI: 10.1109/TWC.2013.010214.130724

[32] BOYD, S., VANDEBBERGHE, L. Convex Optimization. New York (USA): Cambridge University Press, 2004. ISBN: 9780521833783

[33] RAZAVIYAYN, M., SANJABI, M., LUO, Z. Q. Linear transceiver design for interference alignment: Complexity and computation. IEEE Transactions on Information Theory, 2012, vol. 58, no. 5, p. 2896-2910. DOI: 10.1109/TIT.2012.2184909

\section{About the Authors ...}

Ha Hoang KHA received the B.Eng. and M.Eng. degrees from Ho Chi Minh City University of Technology, in 2000 and 2003, respectively, and the Ph.D. degree from the University of New South Wales, Sydney, Australia, in 2009, all in Electrical Engineering and Telecommunications. From 2000 to 2004, he was a research and teaching assistant with the Department of Electrical and Electronics Engineering, Ho Chi Minh City University of Technology. He was a visiting research fellow at the School of Electrical Engineering and Telecommunications, the University of New South Wales, Australia, from 2009 to 2011. He was a postdoctoral research fellow at the Faculty of Engineering and Information Technology, University of Technology Sydney, Australia from 2011 to 2013. He is currently a lecturer at the Faculty of Electrical and Electronics Engineering, Ho Chi Minh City University of Technology, Vietnam. His research interests are in digital signal processing and wireless communications, with a recent emphasis on convex optimization techniques in signal processing for wireless communications. 